\title{
Characteristic Analysis of Sandstorms in Taklamakan Desert
}

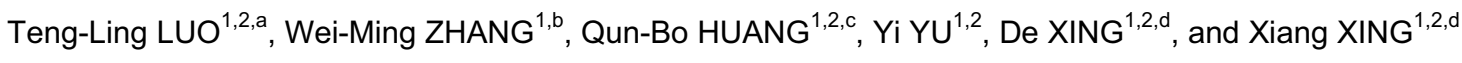 \\ ${ }^{1}$ Academy of Ocean Science and Engineering, National University of Defense Technology, Changsha, China \\ ${ }^{2}$ College of Computer, National University of Defense Technology, Changsha, China \\ aluot117@163.com
}

\begin{abstract}
Firstly, the annual variation of sandstorm and strong sandstorm weather process in China from 2000 to 2012 is analyzed according to the"Sand-Dust Weather Yearbook" (2012). Secondly, based on the ERA-Interim Reanalysis from ECMWF and MISR data from the Terra satellite, we investigate the correlation between different dust weather process and land meteorological elements. Finally, the temporal and spatial distribution features of the aerosol optical depth (AOD) in the Taklamakan Desert is studied. And we compare the Taklamakan Desert AOD with nationwide AOD. The results show that: (1) the frequency of sandstorm and strong sandstorm has shown a downward trend and the occurrence of sandstorm decreases more in recent years. (2) In the Taklamakan Desert, the number of sandstorm is positively correlated with the surface temperature, meanwhile, negatively related to the surface relative humidity. (3) In all seasons, the average of AOD in Taklamakan Desert is higher than that of the whole country, and there are obvious differences among the four seasons.
\end{abstract}

\section{Introduction}

Sandstorm is a weather phenomenon that strong wind blows dust on the ground, making the air turbid and causing the level of visibility less than $1 \mathrm{~km} 1$. When sandstorms happen, sand will be blown up to the troposphere and above under the influence of turbulence or cyclones 23. In the atmosphere, lofted dust is a kind of aerosol which can act as cloud condensation nuclei and ice nuclei. And the dust in the air can scatter and absorb solar radiation, which may influence the radiation energy budget. Besides, Sandstorm can cause huge damage to the society, politics and economy of a country or a district. Nowadays the adverse effects of sandstorm have caught more attention from the Chinese government.

The Taklamakan Desert is the largest desert in China which covers an area of $270000 \mathrm{~km}^{2}$ of the Tarim Basin. As the major source of dust in China, it is necessary to analyze the characteristics of sandstorm in the Taklamakan, in order to provide a theoretical basis for the prevention and control of sandstorm.

Many researchers have done much work about sandstorm in China. Qiu et al. suggested that China's sandstorm mainly occurred in the southern and northern of desert in Xinjiang and its surrounding areas, Gansu Hexi Corridor, dry desert in Inner Mongolia and Qinghai Qaidam Basin and other places 4. Wang et al. analyzed the characteristics of dust circulation in the north of China 5. Wang et al. investigated the path of dust transmission 6 . Shi et al. studied the relationship between the sandstorm in China and the global environment 7. Wang et al. used the climate model to predict the abnormal climate and sandstorm 8.

Since the occurrence of sandstorm is directly affected by the surface meteorological elements, it is essential to find out the relationship between the surface meteorological elements and the frequency of sandstorms. In the paper, we try to discuss the characteristics of sandstorms based on the temporal and spatial distribution features of AOD and the land meteorological elements. We hope to offer a better understanding of the causes of sandstorm in the Taklamakan area.

\section{Data Sets}

In this paper, we have three data sources. The first data is the production of multi-angle imaging spectrometer (MISR) on the satellite "Terra". During the daytime the Terra passes over the equator at 10:45 LT. And the MISR on the Terra has a push-broom camera to measure the Earth at nine different along-track view angles. Besides, MISR can retrieve aerosol properties and it can produce aerosol optical depth (AOD) globally. In this paper we used Level 3 aerosol optical depth (AOD) data from January 2000 to December 2012 with the resolution of $0.5^{\circ}$ by $0.5^{\circ}$.

The second data comes from the ERA-Interim Reanalysis data of ECMWF which provides data from 1979 to the present. With the help of model improvements, Substantial improvement have been archived in ERAInterim Reanalysis data. The ERA-Interim products have a variety of 3-hourly surface parameters, land-surface 
conditions. Here we mainly use the surface temperature and surface relative humidity with the resolution of $1^{\circ}$ by $1^{\circ}$.

The last one is the "Sand-Dust Weather Yearbook" (2012) which is published by China Meteorological Administration. The "Sand-Dust Weather Yearbook" (2012) recorded sandstorms that occurred from 2000 to 2012 in China and provided basic information of sandstorm. In this study, we selected 29 sandstorms from 2000 to 2012 as the research objective to study the characteristics of ground meteorological elements during the period of sandstorms.

\section{The Basic Analysis of Sandstorm in the Taklamakan Desert}

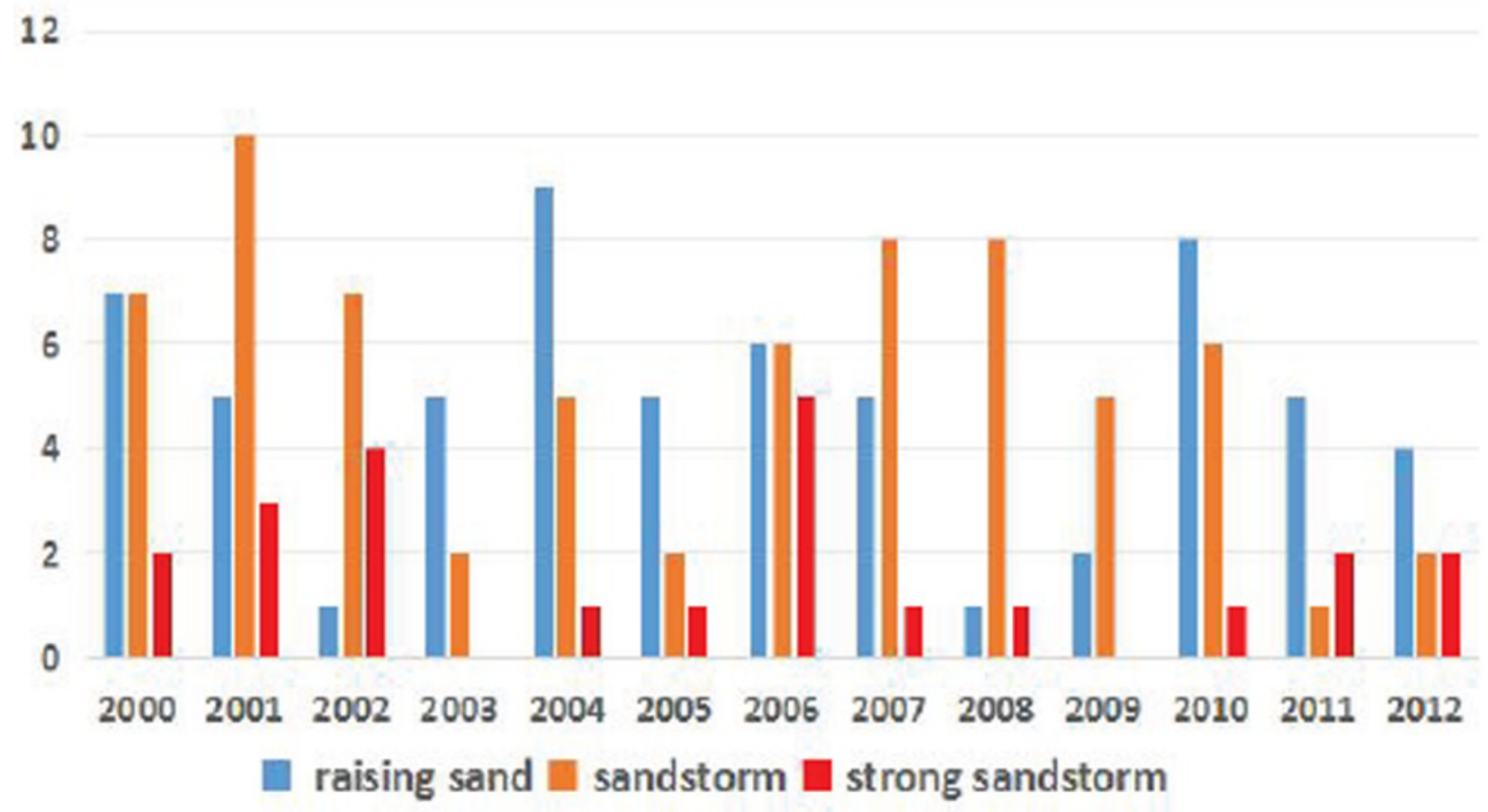

Figure 1. The number of sandstorms occurred in the spring

Figure 1 shows the statistics of sandstorms in the Taklamakan Desert during the spring of $2000 \sim 2012$. The data is picked out from the "Sand-Dust Weather Yearbook" (2012). We record the number of raising sand, sandstorm and strong sandstorm in the Taklamakan Desert. It can be seen that raising sand and sandstorms appear more than strong sandstorm. Raising sand occurs most (9 times) in the year of 2004 while the least occurrence (only once) happens in 2002 and 2008. The sandstorm happens most (10 times) in 2001, but the least number is merely once in 2011. The number of strong sandstorms in 2006 is the highest (5 times), while the least occurs in 2003, and there is no strong sandstorm in this year. 


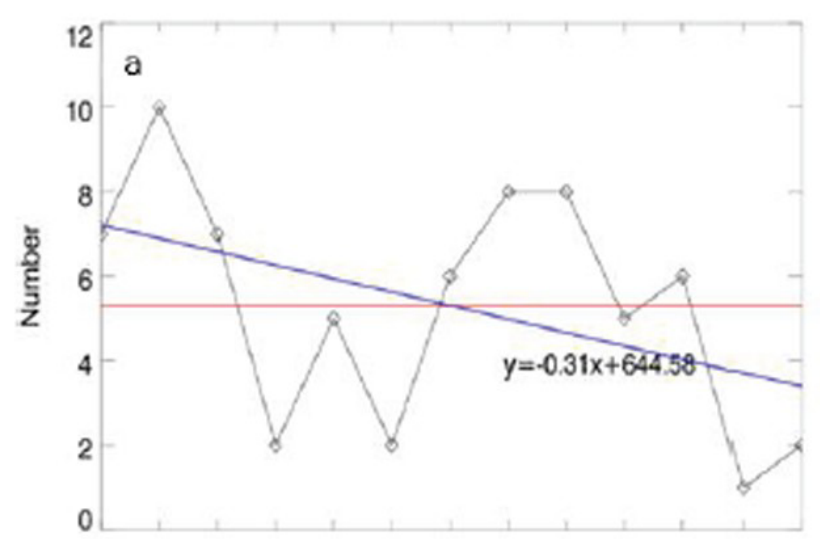

2000200120022003200420052006200720082009201020112012 Year

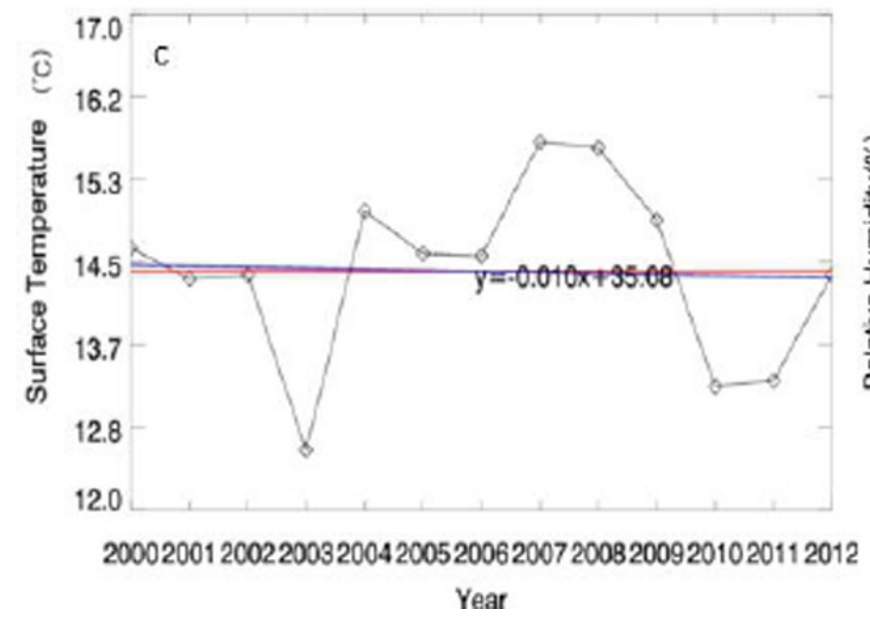

Figure 2. Statistical analysis of changes for the year 2000 through 2012 (a) sandstorm (b) strong sandstorm (c) surface temperature (d) surface relative humidity

Figure $2 \mathrm{a}$ shows a significant decline of the annual occurrence frequency of sandstorm in the Taklamakan Desert. Similarly, there is also a downtrend in the happening of strong sandstorm in Figure 2b. It can easily conclude that the frequency of sandstorm has a larger drop than the strong sandstorm from 2000 to 2012 in the Taklamakan Desert by comparing Figure 2a and Figure $2 b$.

From Figure $2 \mathrm{a}$ and Figure $2 \mathrm{c}$, we can infer a positive relationship between the appearance of sandstorm and the land surface temperature. As we can see, when the surface temperature is higher in the year of 2006 to 2009, the sandstorm also happens more in those years. On the contrary, there are fewer sandstorms from 2002 to 2004 when the surface temperature is lower. The reason of this positive relationship may be that high temperature will produce updrafts, resulting in the decrease of the atmosphere stability and leads to sandstorm.

In the same way, we can infer that there is a negative correlation between the occurrence of sandstorm and the surface relative humidity. From the figure $2 \mathrm{a}$ and Figure $2 \mathrm{~d}$, when the surface relative humidity is higher in the years of 2003, 2004 and 2005, sandstorms happen fewer. However, sandstorms occur more in 2007 and 2008 when the surface relative humidity is lower. This negative correlation may be caused by the fact that the drier of the sand, the easier it will be lofted by wind, which produces the sandstorm.
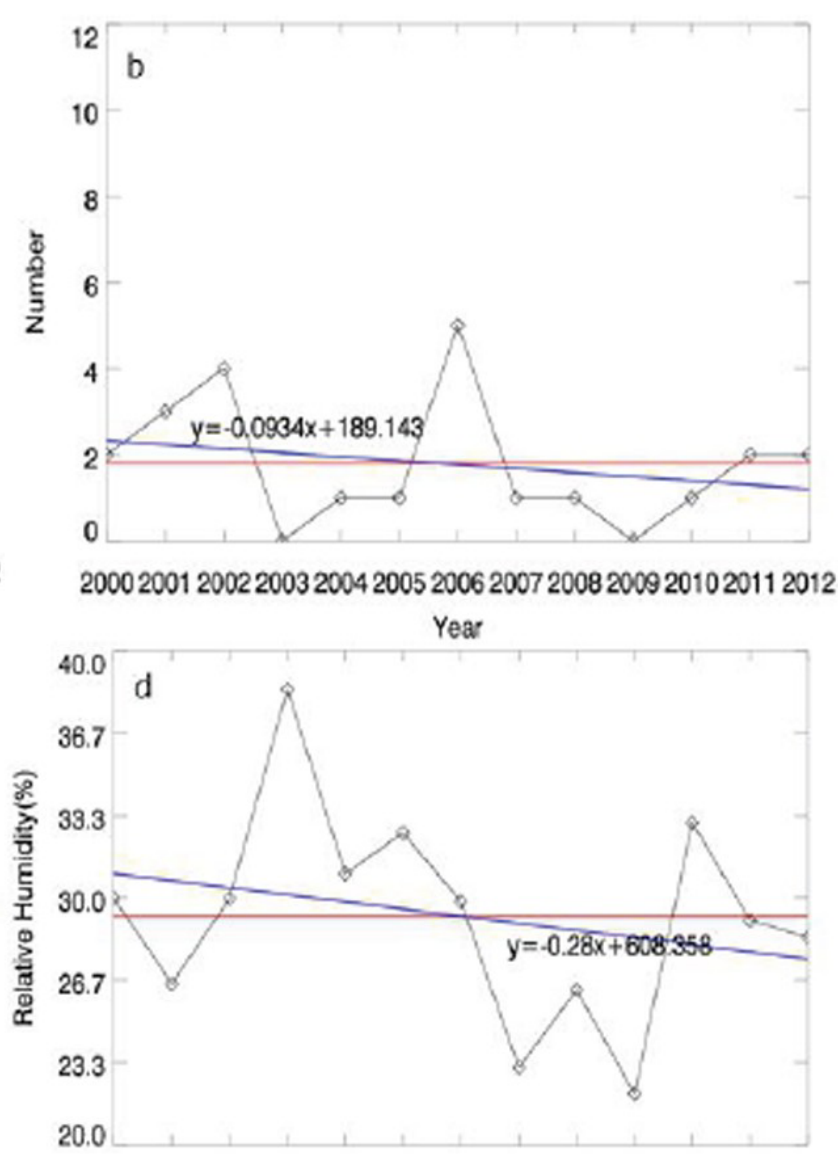

2000200120022003200420052006200720082009201020112012

Year

Table 1 shows the correlation coefficients of two types of sandstorms and the two kinds of surface meteorological elements. According to the data, the correlation coefficient between the frequency of sandstorm and the surface temperature is as high as 0.526 and pass the significant test, which confirms our inference above. Nevertheless, the correlation between the strong sandstorm and land surface temperature is not supported. The coefficient is only 0.044 , which fails the significant test.

Table 1. Coefficient of correlation between meteorological element and type of sandstorm

\begin{tabular}{|c|c|c|}
\hline Type of sandstorm & Sandstorm & Strong sandstorm \\
\hline Meteorological elements & 0.526 & 0.044 \\
\hline Surface temperature & -0.471 & -0.042 \\
\hline
\end{tabular}

The relationship between the frequency of sandstorms and surface relative humidity is also verified, with a high coefficient -0.471 . However, the correlation between the surface relative humidity and the number of strong sandstorms is also not significant, and the correlation coefficient is only -0.042 without passing the significance test. 
The probable reason of the weak link between the surface temperature, surface relative humidity and the strong sandstorms is that the strong sandstorm is mainly affected by the weather process rather than the others.
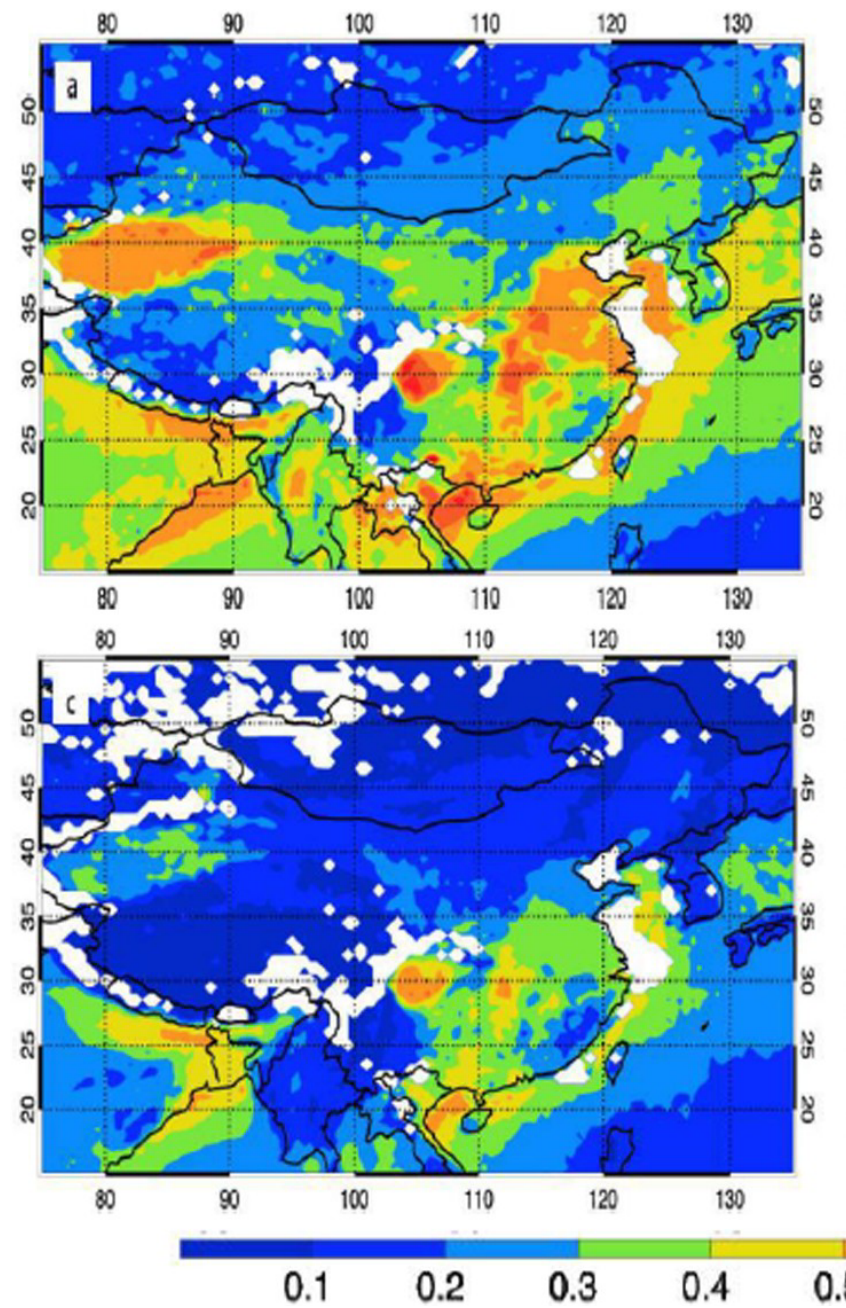

\section{The temporal and spatial distribution features of AOD in the Taklamakan Desert.}
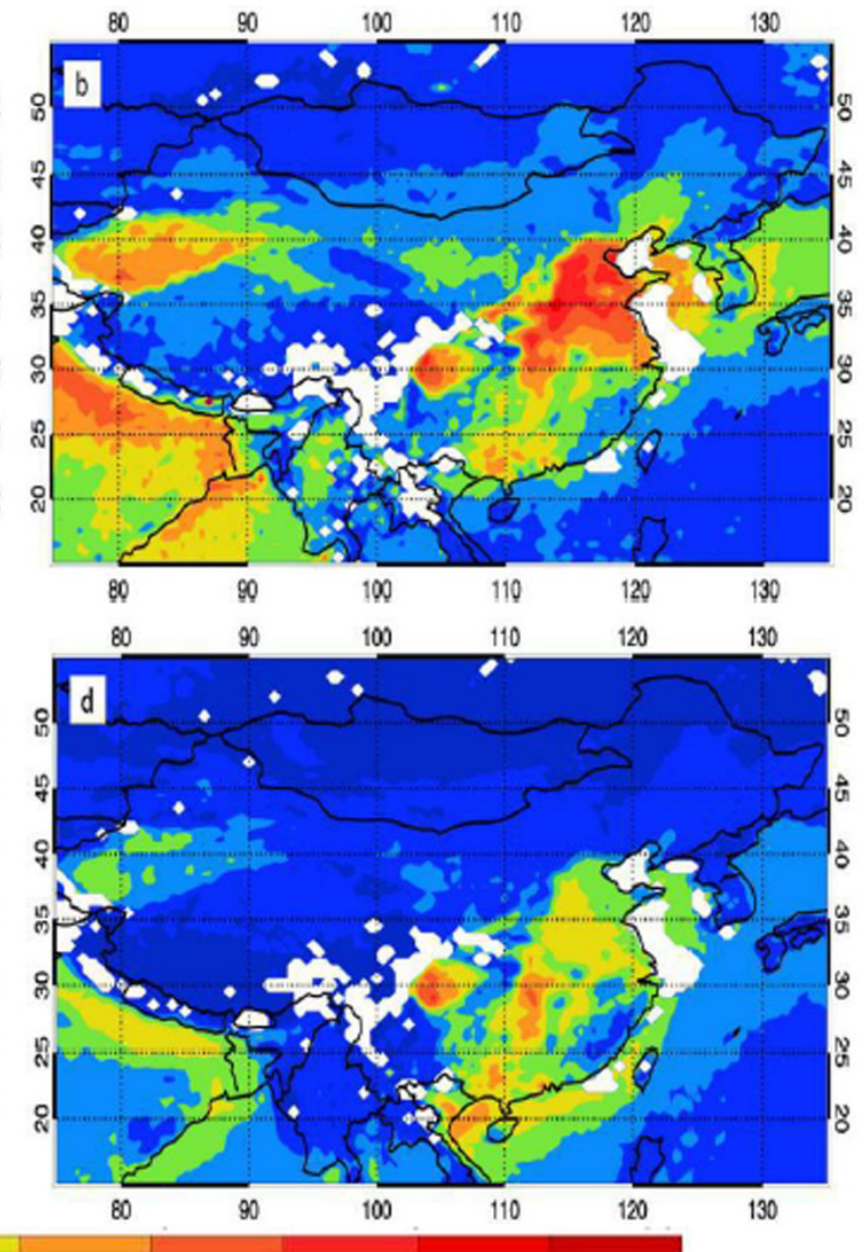

$\begin{array}{llll}0.6 & 0.7 & 0.8 & 0.9\end{array}$

Figure 3. Seasonal mean AOD in China. (a) spring (b) summer (c) autumn (d) winter

Figure 3 shows the temporal and spatial distribution features of AOD in the Taklamakan Desert from 2000 to 2012. In the spring, there is a high value center in the Aksu and Korla areas, north of the Taklamakan Desert and near the Ruoqiang in the east, with a maximum optical thickness of 0.7 . In the summer, the frequency of sandstorms decreases without the effects of cold front. And the value of the overall level of the AOD in summer is lower than that in spring, but there is also a high value (0.7) in the southern part of the Taklamakan Desert. However, in autumn and winter, there is almost no sandstorm. So the optical thickness of the AOD reduces rapidly, with a maximum value of 0.3 .

Overall, there is thicker AOD in the southern and northern regions of the Taklamakan Desert, which means these two areas tend to experience more sandstorms than other places. There may be a reasonable explanation to this phenomenon: the Kunlun Mountain and the Tian Mountain locates in the south and north of the Taklamakan Desert respectively. This kind of terrain may enhance the wind speed on the ground, leading to sandstorms.

\section{Comparative Analysis of Aerosol Optical Depth (AOD) of China and Taklimakan Desert}

We further compared the frequency of AOD in China and the Taklimakan Desert. Figure 4a shows the average AOD of the Taklimakan Desert is 0.4 in the spring, much higher than the average AOD of the China over this period of 0.12 . This phenomenon indicates that sandstorms are strong in the spring in the Taklimakan Desert. Figure $4 \mathrm{~b}$ presents that the average of AOD in the Taklimakan Desert fell to 0.3 in the summer as the frequency of sandstorms decreasing. It is found that the average AOD in the Taklimakan Desert fell again and remained at around 0.2 , and the average of the nationwide AOD remained at around 0.1 , for there is little sandstorm in autumn and winter ( Figure 4c, 4d). 

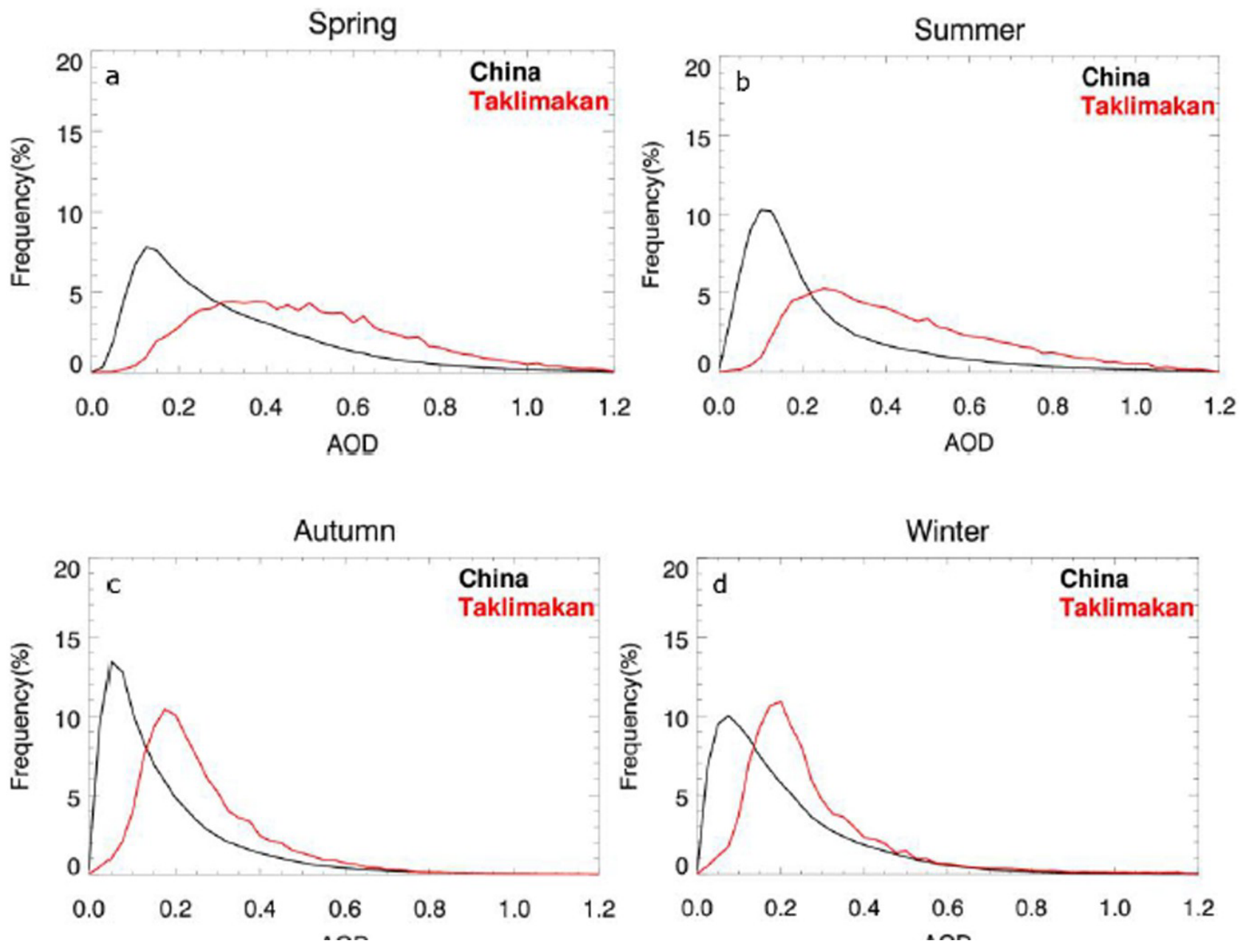

Figure 4. Probability distribution function (PDF) of AOD. (a) spring (b) summer (c) autumn (d) winter

In summary, the AOD in spring and summer is higher than that in autumn and winter, which indicates that sandstorms mainly occur in spring and summer. In all seasons, the average AOD in the Taklimakan Desert is greater than that of the nationwide AOD.

\section{Discussion and Conclusion}

We used the "Sand-Dust Weather Yearbook" to make basic analysis of sandstorms in the Taklamakan desert, and we also used ERA-Interim Reanalysis data to have correlative analysis of meteorological elements and sandstorm. The AOD data of MISR from 2000 to 2012 were used to study the characteristics of AOD in the Taklimakan Desert.

Results show that the frequency of sandstorm and strong sandstorm in recent years has shown a downward trend and the occurrence of sandstorm decreases more. The number of sandstorm is positively correlated with the surface temperature, while negatively related to the relative humidity. MISR data shows that the AOD is higher in the spring and summer than that in the autumn and winter, which indicates that sandstorms occur mainly in spring and summer. In all seasons, the average of AOD in Taklamakan Desert is higher than that of the whole country and the difference among the four seasons is obvious.
Although we have analyzed the correlation among sandstorms and surface temperature and surface relative humidity, we still have a long way to go. There are many other factors affecting sandstorms, such as polar vortex, evaporation rate and precipitation. Besides weather situation, atmospheric stability and wind field characteristics may be also important factors causing to sandstorms, which should also attract our attention in the future.

\section{Acknowledgment}

The work was supported by the Natural Science Foundation of China (Grant No. 41375113).

\section{References}

1. China Meteorological Administration. Sand-Dust Weather Yearbook [M], 2014.6

2. Kaufman, Y. J., D. Tanre, and O. Boucher (2002), A satellite view of aerosols in the climate system, Nature, 419, 215-223.

3. Rosenfeld,D.,Y. Rudich, and R. Lahav(2001),Desertdust suppressing precipitation: A possible desertification feedbackloop, Proc. Natl. Acad.Sci. U.S.A., 98, 5975-5980. 
4. Qiu X F, Zeng Y, Miaq Q L. Temporal-spatial distribution as well as tracks and source areas of sand-dust storms in China $[\mathrm{J}]$. Acta Geographica Sinica, 2001(3).

5. Wang K, Jiang H, Hong W U. Circulation Dynamical Structure in Course of Dust Storm Occurrence in North China in Spring of 2001[J]. Plateau Meteorology, 2002.

6. Wang Shigong, WANG Jinyan, ZHOU Zijiang. Regional Characteristics of Dust Events in China[J]. Acta Geographica Sinica, 2003, 58(2):193-200.
7. Shi Y. Discussion on The Present Climate Change from Warm-day to Warm-wet in Northwest China[J]. Quaternary Sciences, 2003, 23(2):152-164.

8. Wang $\mathrm{H}$, Lang $\mathrm{X}$, Zhou $\mathrm{G}$, et al. A Preliminary Report of the Model Prediction on the Forthcoming Winter and Spring Dust Climate over China[J]. Chinese Journal of Atmospheric Sciences, 2003, 27(1):136-140. 